This is the final peer-reviewed accepted manuscript of:

M. Fabrizio; F. Franchi; B. Lazzari; R. Nibbi - A non-isothermal compressible CahnHilliard fluid model for air pollution phenomena -

Physica D 378-379 (2018), pp. 46-53.

The final published version is available online at: https://doi.org/10.1016/j.physd.2018.05.003

Rights / License:

The terms and conditions for the reuse of this version of the manuscript are specified in the publishing policy. For all terms of use and more information see the publisher's website.

This item was downloaded from IRIS Università di Bologna (https://cris.unibo.it/)

When citing, please refer to the published version. 


\title{
A non-isothermal compressible Cahn-Hilliard fluid model for air pollution phenomena
}

\author{
Mauro Fabrizio ${ }^{\mathrm{a}, *}$, Franca Franchi $^{\mathrm{a}}$, Barbara Lazzari ${ }^{\mathrm{a}}$, Roberta Nibbi ${ }^{\mathrm{a}}$ \\ ${ }^{a}$ Department of Mathematics, University of Bologna, Italy
}

\begin{abstract}
Air pollution is an evident and dangerous contamination form. In particular in this paper, we describe a mathematical model for the study of pollution due to the burning of fuels, close to the earth's surface. So, following the phase-field approach, we consider a mixture of two non-isothermal compressible Euler fluids whose mixing is studied by a generalized Cahn-Hilliard theory under suitable constitutive equations. For this model we show the compatibility with the Laws of Thermodynamics, working with the internal (mechanical, heat and chemical) powers. Finally, we implement our model with non-homogeneous boundary conditions, describing the effect of pollutant emission sources like plumes of smoke from the stacks on the earth surface.
\end{abstract}

Keywords: phase-field approach, Cahn-Hilliard equation, compressible ideal gases, thermal effects, air pollution

2010 MSC: 80A17, 92D40, 91B76

\section{Introduction}

Some pollution phenomena are due to the mixing of the air with gases emitted by land discharges, such as smoke produced by many manufacturing plants. Because of these phenomena, in the atmosphere we observe a mixture of two compressible gases, whose evolution strongly depends on the emissions from the earth's surface (see e.g. [1, 2, 3])

It is well known that air pollution is a long standing problem whose effects can spread all over the world with very harmful consequences on public health $[2,4,5,6]$. We think, for example, to the recent attention of EU governments to place a ban on diesel and petrol cars in the near future in order to fight against the poor air quality problem. Indeed, high emissions of $N O_{x}$ from diesel cars is causing serious issues for many governments.

Several attempts have been performed to understand these phenomena via the development of mathematical models from different frameworks (both continuum and stochastic), producing also interesting numerical calculations and simulations in this area (see e.g. [7, 8, 9, 10]). Application fields include not only environmental physics, but also studies involving the spread of pollutants in a layer of fluid or in soils (see e.g. [11]). Since cities have grown enormously with a lot of novel sources of pollutants (chimneys, machines, motors, ...), these models are to be suitably generalized to make them applicable over wider regions, in such a way to better fit the recently observed data. Moreover we observe that several emission sources enter the system as boundary conditions, thus also the formulation of non-homogeneous boundary conditions becomes a new interesting topic.

Unlike the previous Gaussian plumes approach [12, 13], the model we have studied deals with a nonisothermal mixture of two compressible gases, such as air and smoke or fog and smoke, described as a gas

\footnotetext{
${ }^{*}$ Corresponding author

Email addresses: mauro.fabrizio@unibo.it (Mauro Fabrizio), franca.franchi@unibo.it (Franca Franchi), barbara.lazzari@unibo.it (Barbara Lazzari), roberta.nibbi@unibo.it (Roberta Nibbi) 
mixture with a total mass density and an average velocity field, in the presence of Fourier-type thermal effects. On the other hand the capillary properties are related to a generalized Cahn-Hilliard type balance equation for the mass concentration of one of the two components, here interpreted as an order parameter. Thus, to describe the integration/separation of the two gases, we follow a phase-field pattern and recover a modified Cahn-Hilliard-Euler-Fourier model with an effective non-local pressure, accounting for an additional non-stationary contribution, consistent with the presence of an internal capillary structure. As a consequence, the chemical potential includes a new stretching term, due to the different rarefaction of the two gases to be merged, which turns out to be strategic in the mixing/demixing problem analysis. In this way, this model is able to describe the mixing of different components in relation with the environmental conditions, but also their demixing whenever the external climatic factors change. Indeed these naturally occurring situations have a different impact on the environment, in particular we believe that the most harmful impact is due to the separation phenomena.

So, in addition to the continuity, motion and heat balance equations, the Cahn-Hilliard equation is supposed to be a new field equation to which an internal structural power is associated $[14,15,16,17,18,19]$. Furthermore, the use of this internal power into the First Law of Thermodynamics allows to prove the consistency of this complex model with the Second Law of Thermodynamics in its classical form [20].

The outline of the paper is as follows. Section 2 is devoted to introduce some preliminary notations and definitions. Then in Section 3 we provide the balance and constitutive equations in order to describe the model and select appropriate double-well type (polynomial and logarithmic) potentials to give an immediate description of the phase transition problem. In Section 4 we show that this model is compatible with the thermodynamics, while in Section 5 we present the complete set of evolution equations governing our air pollution model, implemented by non-homogeneous boundary conditions, allowing for pollutant sources from the earth surface to the atmosphere. Finally, the last Section is devoted to some final comments and possible future developments.

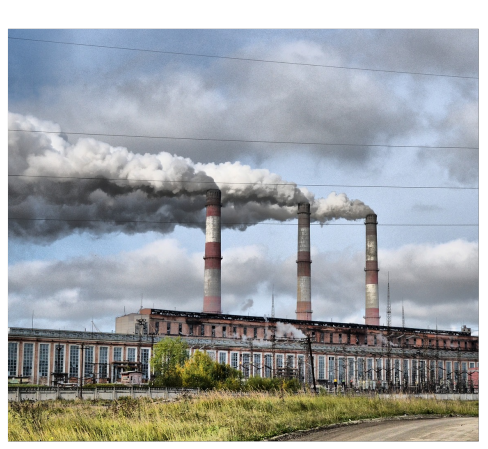

Figure 1: Smoke plumes from stacks.

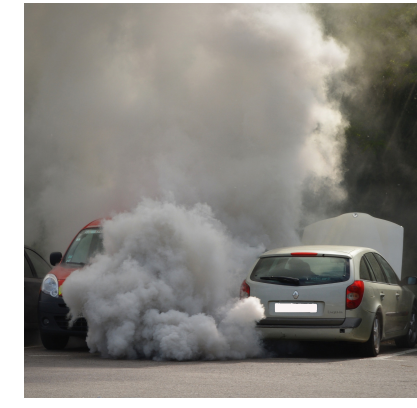

Figure 2: Automobile exhaust gas.

\section{Basic relations for a binary mixture: the phase-field approach}

In order to define a thermodynamically consistent phase-field model for the description of a mixture of two compressible, miscible non-reacting ideal fluids, occupying a possibly time-dependent region $\Omega \subset \mathbb{R}^{3}$, we start with some preliminary definitions.

Denoting by $\rho_{1}$ and $\rho_{2}$ the mass densities of the two constituents, the total mass density of the mixture is given by

$$
\rho=\rho_{1}+\rho_{2} .
$$

Also, let

$$
c=\frac{\rho_{1}}{\rho}=\frac{\rho-\rho_{2}}{\rho}
$$


be the mass concentration of the first constituent in the mixture, representing the (local) order parameter and subject to the condition $0 \leq c \leq 1$, as physically required. Therefore the mass densities $\rho_{1}$ and $\rho_{2}$ are related to $\rho$ and $c$ by

$$
\rho_{1}=c \rho, \quad \rho_{2}=(1-c) \rho .
$$

Henceforth, dealing with the mixture as a whole fluid, we regard $\rho$ and $c$ as independent variables with $c$ playing the role of the phase-field parameter. Indeed the mass densities $\rho, \rho_{1}$ and $\rho_{2}$ and the concentration $c$ are functions of the position $\mathbf{x}$ and time $t$.

In terms of $\rho$ and $c$ the masses of the two constituents in the mixture at time $t$ are expressed as

$$
m_{1}(t)=\int_{\Omega} c(\mathbf{x}, t) \rho(\mathbf{x}, t) d \mathbf{x}, \quad m_{2}(t)=\int_{\Omega}[1-c(\mathbf{x}, t)] \rho(\mathbf{x}, t) d \mathbf{x},
$$

and the mean density of the i-th fluid at time $t$ is

$$
\bar{\rho}_{i}(t)=\frac{m_{i}(t)}{\operatorname{Vol}(\Omega)} \quad i=1,2,
$$

so that

$$
c^{*}(t)=\frac{\bar{\rho}_{1}(t)}{\bar{\rho}_{1}(t)+\bar{\rho}_{2}(t)}
$$

represents the mean value of $c$ at time $t$.

Finally, denoting by $\mathbf{v}_{1}$ and $\mathbf{v}_{2}$ the velocities of the individual constituents, we now define the massaverage (or barycentric velocity) $\mathbf{v}$ as

$$
\mathbf{v}=c \mathbf{v}_{1}+(1-c) \mathbf{v}_{2} .
$$

Obviously the velocities $\mathbf{v}_{1}, \mathbf{v}_{2}$ and $\mathbf{v}$ are functions of $(\mathbf{x}, t) \in \Omega \times(0, T)$.

\section{Balance and constitutive equations}

As just observed, we assume that the binary mixture may be viewed as a single fluid. Then the local balances of mass and linear momentum are written as

$$
\begin{gathered}
\dot{\rho}=-\rho \nabla \cdot \mathbf{v}, \\
\rho \dot{\mathbf{v}}=\nabla \cdot \mathbf{T}+\rho \mathbf{f},
\end{gathered}
$$

where the stress tensor $\mathbf{T}$ is assumed symmetric, while $\mathbf{f}$ is the body force density.

To describe the phase separation problem, we propose the Cahn-Hilliard equation for the concentration as a local balance law:

$$
\rho \dot{c}=-\nabla \cdot \mathbf{J},
$$

$\mathbf{J}$ being the (constitutive) concentration/diffusion flux vector. Hereafter the presence of a supply term is neglected, even if source terms depending on $c$ may be experimentally justified [21].

Also, we introduce the thermal balance law in the standard form (see e.g. [22, 23])

$$
\rho h=-\nabla \cdot \mathbf{q}+\rho r,
$$

where $h$ is the rate at which the heat is absorbed by the mixture, $\mathbf{q}$ is the heat flux vector and $r$ is the heat supply density.

Henceforth we assume that $\mathbf{T}, \mathbf{J}$ and $\mathbf{q}$ are determined by the set $\Gamma$ of the independent variables [24]

$$
\Gamma=(\rho, \theta, c, \nabla \theta, \nabla c) .
$$

through appropriate constitutive functions. 
In agreement with [20], we can say that if the only macroscopic manifestation of internal structures is the order parameter $c$, then it seems reasonable that the interatomic forces may be characterized by macroscopic fields performing work whenever $c$ undergoes changes. Also, in a phase-field model of deformation twinning, surface terms like a flux are to be considered proportional to the order parameter gradient (see e.g.[25]). For these reasons we here take the following split expression for the stress tensor

$$
\mathbf{T}=-p(\rho, \theta, c, \nabla \theta, \nabla c) \mathbf{I}+\mathbf{\Pi},
$$

where the first term represents a generalization of the classical Cauchy stress tensor for a compressible Euler fluid, while $\boldsymbol{\Pi}$ denotes the extra-stress tensor, strictly associated with the non-local capillary properties.

Thinking of (higher order) gradient-flux type constitutive equations, the diffusion flux $\mathbf{J}$ is assumed to be proportional to the gradient of the chemical potential $\mu$, i.e.

$$
\mathbf{J}=-M(\rho, \theta, c) \nabla \mu
$$

where the order parameter mobility $M$ is a nonnegative function and the chemical potential $\mu$, representing the driving force for local and non-local reduction of the concentration within the diffusive interface process, is here generalized as follows (see [26])

$$
\mu(\rho, \theta, c, \nabla c)=f+\frac{1}{2} \frac{\partial}{\partial c} \chi(\rho, \theta, c)|\nabla c|^{2}-\frac{1}{\rho} \nabla \cdot[\rho \chi(\rho, \theta, c) \nabla c],
$$

$\chi$ being the capillary parameter of the standard non-locality due to the concentration gradient. The function $f$ is generally related to the derivative (with respect to $c$ ) of a generalized double-well potential, whose wells correspond to the two bulk phases.

We point out that our previous choices allow for degenerate chemical mobilities, leading to a degenerate nonlinear parabolic type Cahn-Hilliard equation [27].

In order to model air pollution phenomena fairly well, we postulate

$$
f(\rho, \theta, c, \nabla \cdot \mathbf{v})=\theta_{*} F^{\prime}(c)+\left[\theta+\frac{\beta(\rho, \theta) \operatorname{sign}\left(c-c^{*}\right)}{\rho} \nabla \cdot \mathbf{v}\right] G^{\prime}(c),
$$

where $F$ and $G$ are suitable smooth functions depending only on $c$, whose expressions will be specified in the sequel, while $\theta_{*}$ plays the role of a constant critical temperature such that the classical potential

$$
P=F+\frac{\theta}{\theta_{*}} G
$$

exhibits changes in the convexity when $\theta<\theta_{*}$, to reflect the coexistence of the two separated phases; on the contrary for $\theta>\theta_{*}$ the system remains completely mixed.

The additional " $\nabla \cdot \mathbf{v}$-term" in $(7)$, due to the different rarefaction of the two gases to be merged, is strictly related to the mean value $c^{*}$ of the concentration via an arbitrary non-negative scalar valued function $\beta$. Accordingly we propose the following constitutive equation for the extra-stress tensor $\mathbf{\Pi}$,

$$
\boldsymbol{\Pi}=-\beta(\rho, \theta) \operatorname{sign}\left(c-c^{*}\right) \dot{G}(c) \mathbf{I}-\chi(\rho, \theta, c) \nabla c \otimes \nabla c .
$$

The first term of (9) may be interpreted as a (non-stationary) rate-type pressure of the mixture with " $\beta \operatorname{sign}\left(c-c^{*}\right)$ " playing the role of a chemical relaxation time, whereas the second one represents the capillary forces contribution in the standard dyadic form.

As usual, to address the transition problem we restrict our attention to homogeneous regions and stationary conditions and hence $(\rho, \theta, c, \nabla \cdot \mathbf{v})$ are uniform, so that the chemical potential reduces to $(7)$.

For definiteness and in absence of the stretching term, we now give two examples of the potential (8) which allow for an immediate description of the thermally induced phase transition: the typical polynomial and the singular logarithmic ones. 
In the first case we select the functions $F$ and $G$ as follows

$$
F_{1}(c)=\left(c-c^{*}\right)^{4}-\alpha\left(c^{*}\right)\left(c-c^{*}\right)^{2}, \quad G_{1}(c)=\alpha\left(c^{*}\right)\left(c-c^{*}\right)^{2}, \quad c \in[0,1]
$$

where $\alpha\left(c^{*}\right)=2 \min \left\{c^{* 2},\left(1-c^{*}\right)^{2}\right\}$, so that both functions vanish at $c=c^{*}$ along with their first derivatives while, for $c \neq c^{*}, F_{1} \leq 0$ and $G_{1} \geq 0$.

As a result of a very simple qualitative analysis, when $\theta>\theta_{*}$ the potential $P$ admits a (unique) minimum at $c=c^{*}$, i.e. the mixed phase is stable and the two gases are quite merged. On the other hand, if $\theta<\theta_{*}, P$ has a local maximum at $c=c^{*}$ and other two critical points, corresponding to two minima (symmetric with respect to $c^{*}$ ), which means that the mixed phase is unstable and phase separation occurs. Consequently, $\theta_{*}$ plays the role of the transition temperature (see Figure 3 for the plots of $P$ in correspondence of different values of $\theta$ with respect to $\left.\theta_{*}\right)$.

Furthermore, another thermodynamical relevant potential may be given by choosing in (8) the following (singular) logarithmic functions (see e.g. [28, 29, 30]) defined for $c \in(0,1)$ :

$$
F_{2}(c)=2 u(c)(1-u(c)), \quad G_{2}(c)=[u(c) \ln u(c)+(1-u(c)) \ln (1-u(c))]
$$

with

$$
u(c)= \begin{cases}c^{-\frac{\ln 2}{\ln c^{*}}} & \text { if } c^{*}<\frac{1}{2}, \\ c & \text { if } c^{*}=\frac{1}{2}, \\ 1-(1-c)^{-\frac{\ln 2}{\ln \left(1-c^{*}\right)}} & \text { if } c^{*}>\frac{1}{2} .\end{cases}
$$

It is easy to show that, with this second choice, (8) vanishes for $u(c) \rightarrow 0^{+}$and $u(c) \rightarrow 1^{-}$and presents indeed a double-well form under the condition $\theta<\theta_{*}, c=c^{*}$ representing the maximum point. As before, for $\theta>\theta_{*},(8)$ is always a convex function with a minimum at $c=c^{*}$ (see Figure 4 for the plots of $P$ in correspondence with different values of $\theta$ with respect to $\theta_{*}$ ).
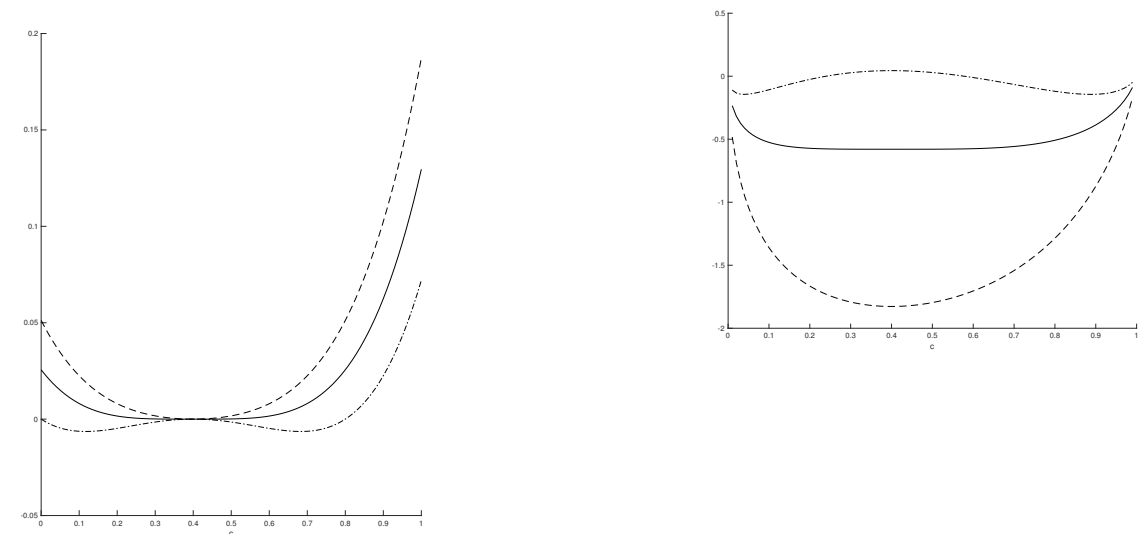

Figure 3: Plot of the polynomial type potential $P$ for $c^{*}=0.4$, in correspondence with $\theta=\theta_{*}$ ( solid

Figure 4: Plot of the logarithmic type potential $P$ for $c^{*}=0.4$, in correspondence with $\theta=\theta_{*}$ (solid line), $\theta>\theta_{*}$ (dashed line), $\theta<\theta_{*}$ (dotdash line). line), $\theta>\theta_{*}$ (dashed line), $\theta<\theta_{*}$ (dotdash line).

By summarizing, phase separation is induced by changes in convexity/concavity in the potential (8) and such changes are related to the passage from minima to maxima or viceversa. To see the mechanical effects on the mixing problem, we now focus on the role of the new stretching term when $\beta$ is a positive constant parameter. To begin we suppose the concentration $c$ within the range $\left(c^{*}, 1\right)$ : it is easy to note that an expanding gas mixture (i.e. $\nabla \cdot \mathbf{v}>0$ ) reinforces the mixing of the two gases. An analogous stabilizing effect is obtained for a compressed gas mixture (i.e. $\nabla \cdot \mathbf{v}<0$ ), when $0<c<c^{*}$. Clearly all other cases favour the demixing of the two gases. So, as experimentally confirmed, a thermally induced phase transition results 
strictly related to the different rarefaction of the two gases together with the range of variability of the concentration with respect its mean value $c^{*}$.

To provide a graphical description of the mechanical effects of the new stretching term, we assume that $\beta, \rho$ and $\nabla \cdot \mathbf{v}$ be constant and, after introducing the following functional

$$
\tilde{P}=F+\frac{1}{\theta_{*}}(\theta G+H)
$$

where

$$
H^{\prime}(c)=\left[\frac{\beta \operatorname{sign}\left(c-c^{*}\right)}{\rho} \nabla \cdot \mathbf{v}\right] G^{\prime}(c),
$$

we plot $\tilde{P}$ within the polynomial choice $(10)$, according to the signs of $\nabla \cdot \mathbf{v}$ and $\left(\theta-\theta_{*}\right)$.
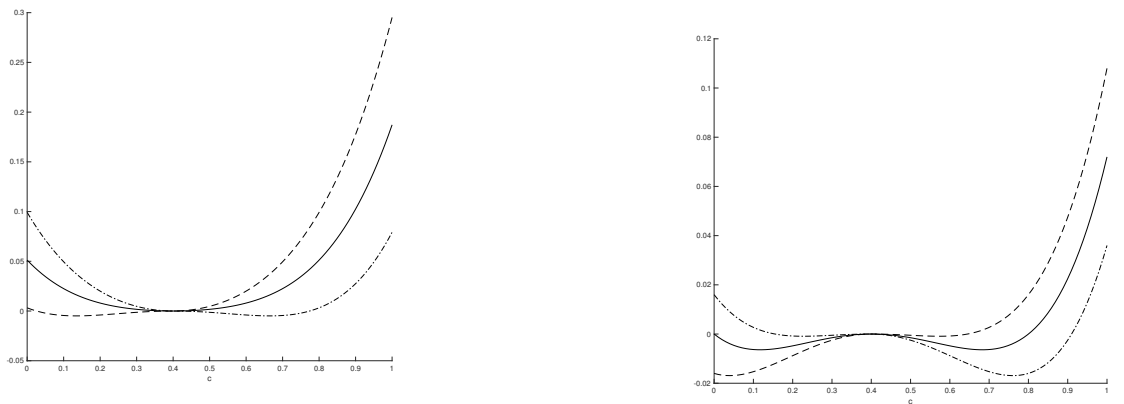

Figure 5: Plot of the polynomial type $\tilde{P}$ for $c^{*}=$ 0.4 , and $\theta>\theta_{*}$, in correspondence with $\nabla \cdot \mathbf{v}=0$ (solid line), $\nabla \cdot \mathbf{v}>0$ (dashed line), $\nabla \cdot \mathbf{v}<0$ (dotdash line).

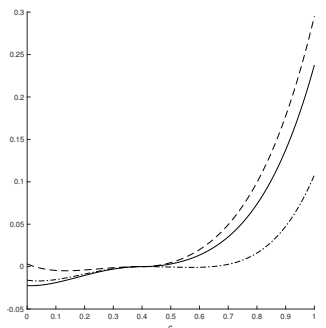

Figure 6: Plot of the polynomial type $\tilde{P}$ for $c^{*}=$ 0.4 , and $\theta<\theta_{*}$, in correspondence with $\nabla \cdot \mathbf{v}=0$ (solid line), $\nabla \cdot \mathbf{v}>0$ (dashed line), $\nabla \cdot \mathbf{v}<0$ (dotdash line).
Figure 7: Plot of the polynomial type $\tilde{P}$ for $c^{*}=$ $0.4, \nabla \cdot \mathbf{v}<0$ in correspondence with $\theta=\theta_{*}$ (solid line), $\theta>\theta_{*}$ (dashed line), $\theta<\theta_{*}$ (dotdash line).

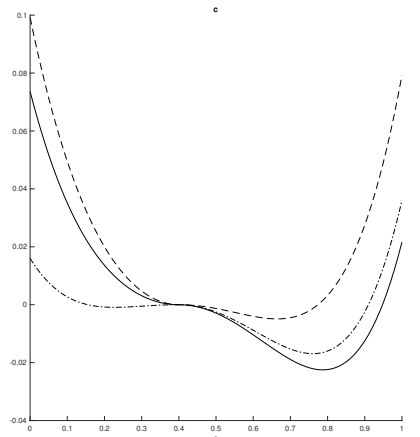

Figure 8: $\quad$ Plot of the polynomial type $\tilde{P}$ for $c^{*}=$ $0.4, \nabla \cdot \mathbf{v}>0$ in correspondence with $\theta=\theta_{*}$ (solid line), $\theta>\theta_{*}$ (dashed line), $\theta<\theta_{*}$ (dotdash line).

Finally, in order to avoid further complications, the temperature field is here incorporated into the energy equation by means of the classical Fourier law of heat conduction, namely the heat flux vector is supposed 
to satisfy the constitutive equation

$$
\mathbf{q}=-k(\rho, \theta, c) \nabla \theta
$$

where the thermal conductivity $k$ is a positive valued scalar function.

Indeed, thinking of global warming as a result of the greenhouse effect and gases emissions, also for these pollution problems one might generalize the heat conduction theory taking into account memory effects and hence allowing for temperature to travel as a wave rather than simply by diffusion (e.g. Cattaneo-Maxwell or Green-Naghdi type 3 theories). In this regard we cite for example [17, 31,32] and the extensive account in the book [33] by Straughan.

\section{Thermodynamic restrictions}

Following classical thermodynamics arguments, besides the internal mechanical and heat powers $\mathcal{P}^{i, m}$ and $\mathcal{P}^{i, h}=\rho h$, we here introduce an internal structural power $\mathcal{P}^{i, c}$ due to the chemical concentration (see e.g. $[34,35]$ and references therein), so that the First Law of Thermodynamics generalizes to the form

$$
\rho \dot{e}=\mathcal{P}^{i, m}+\mathcal{P}^{i, h}+\mathcal{P}^{i, c},
$$

where $e$ is the internal energy of the system. Driven by the non-local capillary properties we have in mind, the internal powers $\mathcal{P}^{i, m}$ and $\mathcal{P}^{i, c}$ are to be chosen as

$$
\begin{aligned}
& \mathcal{P}^{i, m}=-\left[p(\rho, \theta, c, \nabla \theta, \nabla c)+\beta(\rho, \theta) \operatorname{sign}\left(c-c^{*}\right) \dot{G}(c)\right] \nabla \cdot \mathbf{v}-\rho \chi(\rho, \theta, c)(\nabla c \otimes \nabla c) \cdot \nabla \mathbf{v}, \\
& \mathcal{P}^{i, c}= \rho \theta_{*} \dot{F}(c)+\left[\rho \theta+\beta(\rho, \theta) \operatorname{sign}\left(c-c^{*}\right) \nabla \cdot \mathbf{v}\right] \dot{G}(c) \\
&+\frac{1}{2} \rho \frac{\partial}{\partial c} \chi(\rho, \theta, c)|\nabla c|^{2} \dot{c}+\rho \chi(\rho, \theta, c) \nabla c \cdot \nabla \dot{c}+M(\rho, \theta, c) \nabla \mu \cdot \nabla \mu .
\end{aligned}
$$

By means of the identity

$$
\nabla c \cdot \nabla \dot{c}=\overline{(\nabla c)} \cdot \nabla c+(\nabla c \otimes \nabla c) \cdot \nabla \mathbf{v}
$$

it is easy to show that

$$
\begin{aligned}
\mathcal{P}^{i, m}+\mathcal{P}^{i, c}= & p(\rho, \theta, c, \nabla \theta, \nabla c) \frac{\dot{\rho}}{\rho}+\rho \theta_{*} \dot{F}(c)+\rho \theta \dot{G}(c) \\
& +\frac{1}{2} \rho \frac{\partial}{\partial c} \chi(\rho, \theta, c)|\nabla c|^{2} \dot{c}+\frac{1}{2} \rho \chi(\rho, \theta, c) \overline{|\nabla c|^{2}}+M(\rho, \theta, c) \nabla \mu \cdot \nabla \mu .
\end{aligned}
$$

The Second Law of Thermodynamics is taken as the statement that the entropy density $\eta$ satisfies the inequality

$$
\rho \dot{\eta} \geq \rho \frac{h}{\theta}+\frac{\mathbf{q} \cdot \nabla \theta}{\theta^{2}}
$$

for any admissible process compatible with the balance equations (1), (2), (3) and (14). We observe that the usual proportionality relations between the energy and entropy thermal inflows are still preserved.

Hence, substitution of $\rho h=\mathcal{P}^{i, h}$ from (14) and use of the Helmholtz free energy density $\Psi$ defined as $\Psi=e-\theta \eta$, provide the Clausius-Duhem form of the Second Law

$$
\rho(\dot{\psi}+\eta \dot{\theta}) \leq \mathcal{P}^{i, m}+\mathcal{P}^{i, c}-\frac{\mathbf{q} \cdot \nabla \theta}{\theta}
$$

In order to test the thermodynamics consistence of our theory, we let $\Psi$ be given by a $C^{2}$-function of the state variables $\Gamma$. Making use of the chain rule, time differentiation of $\Psi$ and substitution of (17) and (13) into (19) easily yield

$$
\begin{gathered}
{\left[\rho \Psi_{\rho}-\frac{p}{\rho}\right] \dot{\rho}+\rho\left[\Psi_{\theta}+\eta\right] \dot{\theta}+\rho\left[\Psi_{c}-\theta_{*} F^{\prime}(c)-\theta G^{\prime}(c)-\frac{\chi_{c}|\nabla c|^{2}}{2}\right] \dot{c}+\rho \Psi_{\nabla \theta} \cdot \dot{\nabla} \theta} \\
+\rho\left[\Psi_{\nabla c}-\chi \nabla c\right] \cdot \dot{\nabla} c \leq \frac{k \nabla \theta \cdot \nabla \theta}{\theta}+M \nabla \mu \cdot \nabla \mu,
\end{gathered}
$$


where the subscripts stand for the relative partial derivatives.

To begin, for the sake of simplicity, we require that both terms of the right hand side of (20) be nonnegative, which in turn imply the standard restrictions $k \geq 0$ and $M \geq 0$.

Also, the arbitrariness of $\dot{\rho}, \dot{\theta}, \dot{c}, \dot{\nabla \theta}$ and $\dot{\nabla c}$, implies that (20) holds only if

$$
\Psi_{\rho}=\frac{p}{\rho^{2}}, \quad \Psi_{\theta}=-\eta, \quad \Psi_{c}=\theta_{*} F^{\prime}(c)+\theta G^{\prime}(c)+\frac{\chi_{c}|\nabla c|^{2}}{2}, \quad \Psi_{\nabla \theta}=\mathbf{0}, \quad \Psi_{\nabla c}=\chi \nabla c .
$$

Hence it follows that, in compliance with $\Psi$, the pressure $p$ does not depend on $\nabla \theta$ and

$$
p_{\theta}=\rho^{2} \Psi_{\rho \theta}, \quad p_{c}=\rho^{2} \Psi_{\rho c}=\rho^{2} \frac{\chi_{c \rho}|\nabla c|^{2}}{2}, \quad p_{\nabla c}=\rho^{2} \chi_{\rho} \nabla c,
$$

so that $p$ is independent of $c$ if $\chi_{\rho}$ doesn't depend on $c$. In some sense a crucial role is just played by the dependence of the capillarities on $c$.

Therefore, the most general constitutive equation for $p$ may be split as follows

$$
p(\rho, \theta, c, \nabla c)=p_{1}(\rho, \theta)+\frac{1}{2} \rho^{2} \chi_{\rho}(\rho, \theta, c)|\nabla c|^{2},
$$

while the general appropriate form for the Helmholtz free energy $\Psi$ reads

$$
\Psi(\rho, \theta, c, \nabla c)=\psi(\rho, \theta)+\theta_{*} F(c)+\theta G(c)+\frac{1}{2} \chi(\rho, \theta, c)|\nabla c|^{2}
$$

with

$$
\psi_{\rho}=\frac{1}{\rho^{2}} p_{1} .
$$

As a comment we observe that $p_{1}$ stands for the classical thermodynamical pressure, while the other contribution in (23) can be interpreted as the capillary pressure due to inhomogeneous concentration fields.

Consequently to (24), the entropy density $\eta$ is given by

$$
\eta(\rho, \theta, c, \nabla c)=-\psi_{\theta}(\rho, \theta)-G(c)-\frac{1}{2} \chi_{\theta}(\rho, \theta, c)|\nabla c|^{2}
$$

and hence the internal energy assumes the form

$$
e(\rho, \theta, c, \nabla c)=\psi(\rho, \theta)-\theta \psi_{\theta}(\rho, \theta)+\theta_{*} F(c)+\frac{1}{2}\left[\chi(\rho, \theta, c)-\theta \chi_{\theta}(\rho, \theta, c)\right]|\nabla c|^{2} .
$$

In particular

$$
e_{\theta}=-\theta \psi_{\theta \theta}-\frac{1}{2} \theta \chi_{\theta \theta}|\nabla c|^{2}
$$

is the specific heat which generally depends also on $\nabla c$; obviously the (positive) equilibrium specific heat $-\theta \psi_{\theta \theta}$ is recoverable whenever $\chi$ is either a constant parameter or exhibits a (homogeneous or not) linear dependence on $\theta$.

Next, making use of (17), (23), (25) and (27), we reformulate (14) as follows

$$
\begin{aligned}
\rho h & =\rho e_{\rho} \dot{\rho}+\rho e_{\theta} \dot{\theta}+\rho e_{c} \dot{c}+\rho e_{\nabla c} \cdot \dot{\overline{\nabla c}}-\mathcal{P}^{i, m}-\mathcal{P}^{i, c} \\
& =\rho e_{\theta} \dot{\theta}-\rho \theta \Psi_{\theta \rho} \dot{\rho}-\rho \theta\left(G^{\prime}(c)+\frac{\chi_{\theta c}}{2}|\nabla c|^{2}\right) \dot{c}-\frac{\rho}{2} \theta \chi_{\theta} \mid \dot{\left.\nabla \nabla\right|^{2}}-M \nabla \mu \cdot \nabla \mu,
\end{aligned}
$$

so that the heat equation for our theory becomes

$$
\rho e_{\theta} \dot{\theta}+\rho^{2} \theta \Psi_{\theta \rho} \nabla \cdot \mathbf{v}-\rho \theta\left(G^{\prime}(c)+\frac{\chi_{\theta c}}{2}|\nabla c|^{2}\right) \dot{c}-\frac{\rho}{2} \theta \chi_{\theta} \overline{|\nabla c|^{2}}-M \nabla \mu \cdot \nabla \mu=-\nabla \cdot \mathbf{q}+\rho r
$$


where $e_{\theta}$ is specified in (28), while q satisfies (13). It turns out that (30) possesses a strong coupling with other mechanical and capillary aspects, thanks to the dependence of $\chi$ and $k$ on the triplet $(\rho, \theta, c)$.

Finally we highlight that the generalized chemical potential $\mu$ may be written as

$$
\mu=\delta_{c} \Psi+\frac{1}{\rho} \beta(\rho, \theta) \operatorname{sign}\left(c-c^{*}\right) G^{\prime}(c) \nabla \cdot \mathbf{v},
$$

where $\delta_{c} \Psi=\Psi_{c}-\frac{1}{\rho} \nabla \cdot\left(\rho \Psi_{\nabla c}\right)$ represents the variational derivative of the free energy functional, whereas the stretching term reflects and reacts to the introduction of the additional non-stationary average rate-type pressure within the mixture as in [11]. This last term is consistent with the presence of an empirical internal structure due to the mixture, leading to a stronger interlacement between the momentum and Cahn-Hilliard equations.

\section{Evolution equations and boundary conditions}

In this last section, we are now able to present the non-isothermal Cahn-Hilliard-Euler-Fourier system governing the evolution of our air pollution model in $\Omega \times(0, T)$ and then propose appropriate physically admissible boundary conditions. Suppose we now have a half-space $\Omega$ with a fixed lower surface $\partial \Omega$, representing the earth's surface, open to the atmosphere; let $\mathbf{n}$ be its outward unit normal vector.

Thus, by gathering all previous balance and constitutive equations, the general governing system reads as follows

$$
\begin{aligned}
& \dot{\rho}=-\rho \nabla \cdot \mathbf{v}, \\
& \rho \dot{\mathbf{v}}=-\nabla\left[p_{1}(\rho, \theta)+\frac{1}{2} \rho^{2} \chi_{\rho}(\rho, \theta, c)|\nabla c|^{2}+\beta(\rho, \theta) \operatorname{sign}\left(c-c^{*}\right) \dot{G}(c)\right]+ \\
&-\left[\chi(\rho, \theta, c) \Delta c \nabla c+\frac{1}{2} \chi(\rho, \theta, c) \nabla|\nabla c|^{2}+(\nabla \chi(\rho, \theta, c) \cdot \nabla c) \nabla c\right]+\rho \mathbf{f}, \\
& \rho e_{\theta} \dot{\theta}+\rho^{2} \theta \Psi_{\theta \rho} \nabla \cdot \mathbf{v}-\rho \theta\left(G^{\prime}(c)+\frac{\chi_{\theta c}(\rho, \theta, c)}{2}|\nabla c|^{2}\right) \dot{c}-\frac{\rho}{2} \theta \chi_{\theta}(\rho, \theta, c) \mid \dot{\left.\nabla c\right|^{2}} \\
&= M(\rho, \theta, c) \nabla \mu \cdot \nabla \mu+k(\rho, \theta, c) \Delta \theta+\nabla k(\rho, \theta, c) \cdot \nabla \theta+\rho r, \\
& \rho \dot{c}= \nabla \cdot\left\{M ( \rho , \theta , c ) \nabla \left[\theta_{*} F^{\prime}(c)+\left(\theta+\frac{\beta(\rho, \theta) \operatorname{sign}\left(c-c^{*}\right)}{\rho} \nabla \cdot \mathbf{v}\right) G^{\prime}(c)+\frac{1}{2} \chi_{c}(\rho, \theta, c)|\nabla c|^{2}\right.\right. \\
&\left.\left.-\frac{1}{\rho} \nabla \cdot(\rho \chi(\rho, \theta, c) \nabla c)\right]\right\}
\end{aligned}
$$

for $(\mathbf{x}, t) \in \Omega \times(0, T)$, to which we associate prescribed initial conditions for the unknown variables $\rho, \mathbf{v}, \theta$ and $c$. It is evident that if the capillarities are independent of $\rho$ and $c$, as in [24] where $\chi=\chi_{0} \theta$, or even constant, the constitutive setting simplify very much, but we would attain a weaker coupled system for air pollution phenomena, which is less appropriate to fit naturally occurring situations. On the other hand, in our generalization to non-constant parameters, it is just the dependence on $c$ to be responsible in penalizing sharp concentration gradients: this happens through the presence of higher order nonlinearities within the capillarity force.

As for the boundary conditions, it needs to precise that the Cahn-Hilliard theory has been extensively investigated under homogeneous Neumann boundary conditions on $c$ and $\mu$, ensuring that there is no mass diffusion through the boundary, along with standard boundary conditions for the thermo-mechanical frame. More recently, driven by physical considerations, several authors see e.g. [36, 37, 38] have introduced new nonstationary boundary conditions, known as dynamic boundary conditions, allowing for a better exploration of the mixing kinetics of the two state phases. So, the formulation of new non-homogeneous boundary conditions, strictly related to naturally occurring phenomena, becomes another interesting issue within the Cahn-Hilliard theory, both mathematically and empirically.

In the light of the recent boundary proposals, also thinking of the experimental requirements concerning the strong inflow of smoke plumes from stacks (see, for example, figure 1 below), we may simulate that 
pollutant emissions enter the system through the boundary. So we herein account for possible surface sources by assuming the following non-homogeneous boundary conditions on $\partial \Omega \times(0, T)$

$$
\rho(\mathbf{x}, t) \mathbf{v}(\mathbf{x}, t) \cdot \mathbf{n}(\mathbf{x})=g_{1}(\mathbf{x}, t), \quad \nabla \mu(\mathbf{x}, t) \cdot \mathbf{n}(\mathbf{x})=g_{2}(\mathbf{x}, t),
$$

together with non-homogeneous Robin-type boundary conditions for both concentration and temperature, which can an be interpreted as the linearization of Stefan's radiation laws

$$
\nabla c(\mathbf{x}, t) \cdot \mathbf{n}(\mathbf{x})+\alpha_{1} c(\mathbf{x}, t)=g_{3}(\mathbf{x}, t), \quad \nabla \theta(\mathbf{x}, t) \cdot \mathbf{n}(\mathbf{x})+\alpha_{2} \theta(\mathbf{x}, t)=g_{4}(\mathbf{x}, t),
$$

where $\alpha_{1}, \alpha_{2}$ are positive constants, while $g_{1}, g_{2}, g_{3}$ and $g_{4}$ are prescribed functions with $g_{1} \leq 0$. This model may be further specialized to the thermal convection problem in a (multy-)layer heated from below, in order to investigate convectively unstable motions. This situation is peculiarly beneficial in air pollution studies over large urban areas, where a lot of sources of pollutants are present.

\section{Conclusions}

Air pollution is an age-old topic whose newness is nowadays strictly related to the environmental degradation, due to poor air quality with significant health effects on living organisms all over the world. It represents an evident and dangerous contamination form and may occur, for example, owing to the excessive burning of fossil fuels, chimney stacks, biomass incineration, etc. Several attempts have been performed to understand it and fight against these phenomena, also through the development of mathematical modelings from different backgrounds.

In order to gain a better understanding of dispersion and reaction of pollutants, we are primarily interested in the interactions between two gases like air and smoke (or fog and smoke). So, following the phase-field approach, we here present a novel non-isothermal air pollution model within a modified CahnHilliard theory in a binary mixture of two gases, combined with an adequate thermo-mechanical setting. Interestingly, this model results to be compatible with the Laws of Thermodynamics.

As for the boundary conditions, we take into account the effects of the pollution near the earth surface and, differently from other authors, we propose novel non-homogeneous boundary conditions to account for the emissions of smoke from chimney stacks.

The model may be also easily generalized to include reaction terms, like a concentration source density, within the Cahn-Hilliard equation in order to better fit real life situations. Also, a different constitutive thermal setting may be important in interpreting the role of temperature on pollution phenomena.

Finally, as many application areas for air pollution include anisotropic situations, a particular interest may be addressed in the future to cases where the mobilities and capillarities are anisotropic and can be described by non-constant symmetric tensors.

\section{Acknowledgments}

Research performed under the auspices of G.N.F.M. - I.N.d.A.M. and partially supported by Italian M.I.U.R.. We would like to thank the referees for their incisive remarks which have led to improvements in the paper.

\section{References}

[1] A. Aggarwal, A. K. Haritash, K. G., Air pollution modelling - A review, Int. J. of Adv. Tech. in Eng. Sc. 2 (6) (2014) $335-364$

DOI www.ijates.com/images/short_pdf/1404134107_P355-364.pdf

[2] A. Louie, R. Pierce, Mathematical models of human exposure to air pollutants, Math Comput Model 10 (1) (1988) 49-64. DOI https://doi.org/10.1016/0895-7177(88)90121-5

[3] G. J. McRae, J. H. Seinfeld, Development of a second-generation mathematical model for urban air pollution-II. evaluation of model performance, Atmos. Environ. 17 (3) (1983) 501-522. DOI https://doi.org/10.1016/0004-6981(83)90124-5 
[4] F. Kelly, J. C. Fussell, Air pollution and public health: emerging hazards and improved understanding of risk, Environ. Geochem. Health 37 (4) (2015) 631-649. doi:10.1007/s10653-015-9720-1.

DOI https://doi.org/10.1007/s10653-015-9720-1

[5] P. M. Mannucci, M. Franchini, Health effects of ambient air pollution in developing countries, Int J Environ Res Public Health 14 (1048). DOI https://doi.org/10.3390/ijerph14091048

[6] R. F. Phalen, The particulate air pollution controversy, Nonlinearity in biology, toxicology, medicine 2 (4) (2004) $259-292$. DOI https://doi.org/10.1080/15401420490900245

[7] J. F. Blowey, C. M. Elliott, The cahn-hilliard gradient theory for phase separation with non-smooth free energy part ii: Numerical analysis, European Journal of Applied Mathematics 3 (2) (1992) 147-179. doi:10.1017/S0956792500000759. DOI http://dx.doi.org/10.1017/S0956792500000759

[8] M. I. M. Copetti, C. M. Elliott, Numerical analysis of the cahn-hilliard equation with a logarithmic free energy, Numerische Mathematik 63 (1) (1992) 39-65. doi:10.1007/BF01385847.

DOI https://doi.org/10.1007/BF01385847

[9] C. M. Elliott, D. A. French, Numerical studies of the cahn-hilliard equation for phase separation, IMA Journal of Applied Mathematics 38 (96-128). DOI https://doi.org/10.1093/imamat/38.2.97

[10] R. Schaefer, M. Smołka, L. Dalcin, M. Paszyński, A new time integration scheme for cahn-hilliard equations, Procedia Computer Science 51 (2015) 1003 - 1012, international Conference On Computational Science, ICCS 2015. doi:https: //doi.org/10.1016/j.procs.2015.05.244.

DOI http://www.sciencedirect.com/science/article/pii/S1877050915010522

[11] F. Franchi, B. Straughan, A comparison of the graffi and kazhikhov-smagulov models for top heavy pollution instability, Adv Water Resour. 24 (6) (2001) 585-594. doi:10.1016/S0309-1708(00)00073-7. DOI https://doi.org/10.1016/S0309-1708(00)00073-7

[12] A. Desalu, L. Gould, F. Schweppe, Dynamic estimation of air pollution, IEEE T AUTOMAT CONTR 19 (6) (1974) 904 $-910$.

DOI https://doi.org/10.1109/TAC.1974.1100742

[13] R. M. El-Kilani, M. H. Belal, Modelling an environmental pollutant transport from the stacks to and through the soil, Journal of Advanced Research 1 (3) (2010) 243-253.

DOI https://doi.org/10.1016/j.jare.2010.05.009

[14] J. W. Cahn, J. E. Hilliard, Free energy of a nonuniform system. I. interfacial free energy, J. Chemi. Phys. 28 (2) (1958) $258-267$. DOI http://dx.doi.org/10.1063/1.1744102

[15] M. Fabrizio, C. Giorgi, A. Morro, Phase separation in quasi-incompressible Cahn-Hilliard fluids, Eur. J. Mech. B Fluids 30 (3) (2011) 281-287. doi:10.1016/j.euromechflu.2010.12.003. DOI http://dx.doi.org/10.1016/j.euromechflu.2010.12.003

[16] M. Fabrizio, D. Grandi, L. Molari, Water evaporation and condensation by a phase-field model, J. Non-Equilib. Thermodyn. 41 (4) (2016) 295-312.

DOI https://doi.org/10.1515/jnet-2016-0004

[17] F. Franchi, B. Lazzari, R. Nibbi, Mathematical models for the non-isothermal Johnson-Segalman viscoelasticity in porous media: stability and wave propagation, Math. Methods Appl. Sci. 38 (17) (2015) 4075-4087. doi:10.1002/mma.3348. DOI http://dx.doi.org/10.1002/mma.3348

[18] M. E. Gurtin, On a nonequilibrium thermodynamics of capillarity and phase, Quart. Appl. Math. 47 (1) (1989) $129-145$. doi:10.1090/qam/987902. DOI http://dx.doi.org/10.1090/qam/987902

[19] J. Lowengrub, L. Truskinovsky, Quasi-incompressible Cahn-Hilliard fluids and topological transitions, R. Soc. Lond. Proc. Ser. A Math. Phys. Eng. Sci. 454 (1978) (1998) 2617-2654. doi:10.1098/rspa.1998.0273. DOI http://dx.doi.org/10.1098/rspa.1998.0273

[20] M. E. Gurtin, Generalized Ginzburg-Landau and Cahn-Hilliard equations based on a microforce balance, Phys. D 92 (3-4) (1996) 178-192. doi:10.1016/0167-2789(95)00173-5. DOI http://dx.doi.org/10.1016/0167-2789(95) 00173-5

[21] F. Della Porta, M. Grasselli, Convective nonlocal Cahn-Hilliard equations with reaction terms, Discrete Contin. Dyn. Syst. Ser. B 20 (5) (2015) 1529-1553. DOI https://doi.org/10.3934/dcdsb.2015.20.1529

[22] B. D. Coleman, D. R. Owen, A mathematical foundation for thermodynamics, Arch. Rational Mech. Anal. 54 (1974) 1-104. doi:10.1007/BF00251256. DOI http://dx.doi.org/10.1007/BF00251256

[23] M. Frémond, Non-smooth thermomechanics, Springer-Verlag, Berlin, 2002. doi:10.1007/978-3-662-04800-9. DOI http://dx.doi.org/10.1007/978-3-662-04800-9

[24] M. Fabrizio, C. Giorgi, A. Morro, Phase transition and separation in compressible Cahn-Hilliard fluids, Discrete Contin. Dyn. Syst. Ser. B 19 (1) (2014) 73-88. doi:10.3934/dcdsb.2014.19.73. DOI http://dx.doi.org/10.3934/dcdsb.2014.19.73

[25] J. D. Clayton, J. Knap, A phase field model of deformation twinning: nonlinear theory and numerical simulations, Phys. D 240 (9-10) (2011) 841-858. doi:10.1016/j.physd.2010.12.012. DOI http://dx.doi.org/10.1016/j.physd.2010.12.012 
[26] V. M. Kendon, M. E. Cates, I. Pagonabarraga, J. C. Desplat, P. Blandon, Inertial effects in three-dimensional spinodal decomposition of a symmetric binary fluid mixture: a lattice boltzmann study, Journal of Fluid Mechanics 440 (2001) 147-203. doi:10.1017/S0022112001004682. DOI https://doi.org/10.1017/S0022112001004682

[27] J. W. Barrett, J. F. Blowey, H. Garcke, Finite element approximation of the Cahn-Hilliard equation with degenerate mobility, SIAM J. Numer. Anal. 37 (1) (1999) 286-318. doi:10.1137/S0036142997331669. DOI https://doi.org/10.1137/S0036142997331669

[28] J. W. Cahn, On spinodal decomposition, Acta Met. 9 (1961) 795-801. DOI https://doi.org/10.1016/0001-6160(61)90182-1

[29] L. Cherfils, A. Miranville, S. Zelik, The Cahn-Hilliard equation with logarithmic potentials, Milan J. Math. 79 (2) (2011) $561-596$.

DOI https://doi.org/10.1007/s00032-011-0165-4

[30] R. H. Stogner, G. F. Carey, B. T. Murray, Approximation of Cahn-Hilliard diffuse interface models using parallel adaptive mesh refinement and coarsening with $C^{1}$ elements, Internat. J. Numer. Methods Engrg. 76 (5) (2008) 636-661. DOI https://doi.org/10.1002/nme.2337

[31] A. Berti, I. Bochicchio, M. Fabrizio, Phase separation in quasi-incompressible fluids: Cahn-Hilliard model in the CattaneoMaxwell framework, Z. Angew. Math. Phys. 66 (1) (2015) 135-147. doi:10.1007/s00033-013-0395-0. DOI http://dx.doi.org/10.1007/s00033-013-0395-0

[32] M. Fabrizio, F. Franchi, B. Straughan, On a model for thermo-poroacoustic waves, Internat. J. Engrg. Sci. 46 (8) (2008) 790-798. doi:10.1016/j.ijengsci.2008.01.016.

DOI https://doi.org/10.1016/j.ijengsci.2008.01.016

[33] B. Straughan, Heat waves, Vol. 177 of Applied Mathematical Sciences, Springer, New York, 2011. doi:10.1007/ 978-1-4614-0493-4. DOI https://doi.org/10.1007/978-1-4614-0493-4

[34] A. Berti, I. Bochicchio, A mathematical model for phase separation: a generalized Cahn-Hilliard equation, Math. Methods Appl. Sci. 34 (10) (2011) 1193-1201. doi:10.1002/mma.1432. DOI http://dx.doi.org/10.1002/mma.1432

[35] M. Fabrizio, B. Lazzari, R. Nibbi, Thermodynamics of non-local materials: extra fluxes and internal powers, Contin. Mech. Thermodyn. 23 (6) (2011) 509-525. doi:10.1007/s00161-011-0193-x. DOI http://dx.doi.org/10.1007/s00161-011-0193-x

[36] R. Racke, S. Zheng, The Cahn-Hilliard equation with dynamic boundary conditions, Adv. Differential Equations 8 (1) (2003) 83-110. DOI https://projecteuclid.org/euclid.ade/1355926869

[37] P. Colli, T. Fukao, Cahn-Hilliard equation with dynamic boundary conditions and mass constraint on the boundary, J. Math. Anal. Appl. 429 (2) (2015) 1190-1213. DOI https://doi.org/10.1016/j.jmaa.2015.04.057

[38] P. Colli, T. Fukao, Equation and dynamic boundary condition of Cahn-Hilliard type with singular potentials, Nonlinear Anal. 127 (2015) 413-433.

DOI https://doi.org/10.1016/j.na.2015.07.011 